\title{
BEAM POSITION MONITORS FOR DUKE FEL STORAGE RING*
}

\author{
P. Wang\#, N. Hower, V. Litvinenko, M. Moallem, O. Oakeley, G. Swift, Y. Wu \\ Duke University, Free Electron Laser Laboratory, Department of Physics, NC27708
}

\begin{abstract}
The Duke FEL storage ring is a $1 \mathrm{Gev}$ electron ring, which is designed for driving UV-VUV free electron lasers. The ring has been in operation since November of 1994 [1] but the beam position monitors (BPMs) were connected and operated just recently. The BPM pick-ups are 4 stripline electrodes. In order to reduce the higher-order-mode loss excited by the stored beam at the BPM pick-up area, the BPM vacuum chamber is designed with 4 grounding strips between the electrodes that have the same diameters as the electrode. This design allows the electron beam to see a much smoother vacuum chamber at the BPM area. The pick-up signals are processed by Bergoz's electronic modules, which give X/Y outputs directly. Each BPM has its own process module and 34 modules have been connected to the EPICS control system. The beam orbit now can be displayed and corrected through EPICS in the control room. The system performance and the test data will be presented in this paper.
\end{abstract}

\section{INTRODUCTION}

The Duke FEL storage ring has two arc sections with 6.7meter radii and two long straight sections (34-meter each). The stored beam has a bunch length FWHM (Full Width Half Maximum) of 10-30 mm. The stripline electrode BPM has been developed and installed. Since the vacuum chamber in the arc sections has a much smaller diameter than in the straight sections, two types of BPM electrodes are designed to fit the vacuum chamber size in these areas. A special design effort of adding four grounding strips, which are parallel to the pick-up electrode, has reduced the chamber impedance further. There are 36 BPMs installed on the arc sections, one BPM per arc quadruple magnet, and 18 BPMs in the straight sections. The signal processing system works at $178.5 \mathrm{MHz}$, which is the fundamental ring RF frequency. A total of 34 BPMs have been connected to the EPICS control system. The system has been in operation since September 1998. Detailed mechanical design, calibration procedure and system performance will be presented in the following sections.

\section{MECHANICAL DESIGN}

Both types of BPMs utilize short stripline-style electrodes,

*Work supported by U. S. Air Force Office of Scientific Research Grant F49620-93-1-0590 and U. S. Office of Naval Research Grant N0001494-1-0818

\#Email: wang@fel.duke.edu electrically grounded at one end by either welding or brazing to the outer vacuum envelope, and at the other end separated from making electrical contact by the presence of a physical gap of $0.5-\mathrm{mm}$ width. At the end closest to this gap, a ceramic-to-metal electrical feedthrough is affixed by a lightly welded contact, and the signal induced on the electrode by the passing electron bunch is carried through the vacuum wall to a SMA coaxial connector. The lengths of these striplines (from their grounded end to the center of the electrical feedthrough pin at the other end) are $21.64 \mathrm{~mm}$ for the arc BPM and $30.53 \mathrm{~mm}$ for the straight section BPM. Longer striplines usually are preferred but the available physical space limits it. The inner radii of the striplines in both cases is the same as that of the adjacent vacuum chamber wall, R17.78 $\mathrm{mm}$ for the arc BPM and R47.75 $\mathrm{mm}$ for the straight section BPM. To further enhance the impedance matching, the four electrodes are interleaved with four parallel and otherwise geometrically identical striplines, dubbed by us as "grounding strips", which are brazed or welded to the adjacent tube wall at both ends. The modules are rotated at installation so that the electrode strips are centered at 45 degrees above and below the horizontal orbital plane of the electrons, so as to avoid being struck directly by synchrotron radiation.

A prototype electrode ring with grounding strips was machined from aluminium and the impedance of striplines was measured. With adding of grounding striplines, the impedance is much smaller than the one calculated from a parallel stripline with the same width and space (32-ohm instead of 50-ohm). Adjustments were deduced on the body radii with the goal of achieving 50ohm impedance in each case. The final electrode/grounding strip pieces were machined from onepiece stainless steel cylindrical shells, slit lengthways over part of their length. The critical dimensions on all units were machined to within .001 to .002 inch of their nominal values. Fig. 1 is a photograph of both BPMs.

\section{BENCH CALIBRATION}

After the BPMs were assembled and welded together, they were serialized and individually calibrated. Two kinds of calibrations were performed. One is to measure the offset between the electrical center and the mechanical center. This has been done for every single BPM body and is a very good way of quality control for manufacturing. Another calibration we did is to measure the sensitivity of the electrode to beam motion. This is done for each type of BPM. A bench test set has been 
fabricated to perform these jobs. The test set has a Vblock fixture to hold the BPM body. To simulate the beam, an antenna rod carries the electronic signal going through the BPM chamber to a dummy load. The BPM becomes part of a coaxial transmission line where the rod is the central conductor and the BPM body is the outer conductor. Two tapered cones connected at both ends of the BPM chamber are made to reduce the effects of the impedance mismatch. The output signals from 4 pick-ups are used to get the position of the antenna rod relative to the electrical center of the BPM. The test set is sketched as bellow:

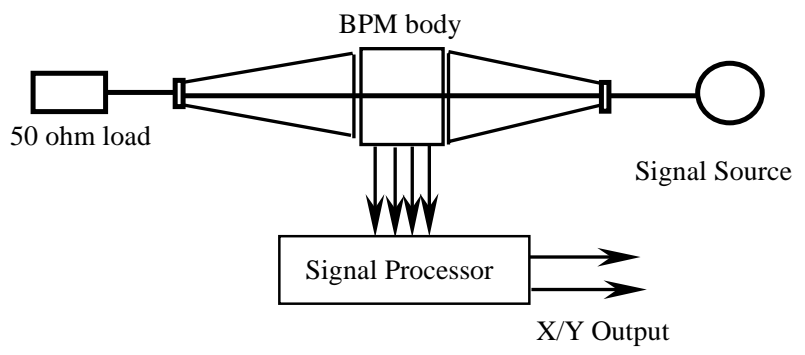

The offset between the electrical center and the mechanical center can be derived from the test data measured with rotating the BPM body. The mechanical center is referred to the outer surface of the BPM body, which has four tooling balls for alignment purposes. We measured about 60 BPM pick-ups of the arc sections and 25 of the straight sections; the offset for most of them is less than 100 micrometers. The sensitivity coefficient is measured with the antenna's displacement near the center. The test results is:

$\mathrm{Sx}=\mathrm{Sy}=6.67 \% / \mathrm{mm}$, for the arc section $\mathrm{Sx}=\mathrm{Sy}=2.8 \% / \mathrm{mm}$, for the straight section

The beam position is determined by the following equations:

$$
\begin{aligned}
& \mathrm{X}=\mathrm{k}^{*}(\mathrm{Va}-\mathrm{Vb}-\mathrm{Vc}+\mathrm{Vd}) /(\mathrm{Va}+\mathrm{Vb}+\mathrm{Vc}+\mathrm{Vd}) \\
& \mathrm{Y}=\mathrm{k}^{*}(\mathrm{Va}+\mathrm{Vb}-\mathrm{Vc}-\mathrm{Vd}) /(\mathrm{Va}+\mathrm{Vb}+\mathrm{Vc}+\mathrm{Vd})
\end{aligned}
$$

Were $\mathrm{Va}, \mathrm{Vb}, \mathrm{Vc}$, and $\mathrm{Vd}$ are detected signals from the pick-up electrodes and $\mathrm{k}=1 / \mathrm{Sx}$.

At the time we did the calibration, the BPM electronics for processing the pick up signals was not available. A short pulse signal generated from the snap diode is used to simulate the bunched beam. The signal's FWHM is around 100ps, which corresponds a bunch length of $30 \mathrm{~mm}$ for a relativistic beam. A fast digital scope, Tektronix DSA-602, is used to record the output signals from each pick-up electrode. Now with a signal processing electronics working at $178.5 \mathrm{MHz}$, we plan to recheck the sensitivity with a $178.5 \mathrm{MHz} \mathrm{CW}$ signal in the near future. The available electrostatic analysis program also will be used to get the sensitivities at larger displacement from the center. By doing so, the pincushion-like distortion can be compensated. According to Jim Hinkson at LBL, the calculated sensitivities have very good agreement with his bench test data [2].

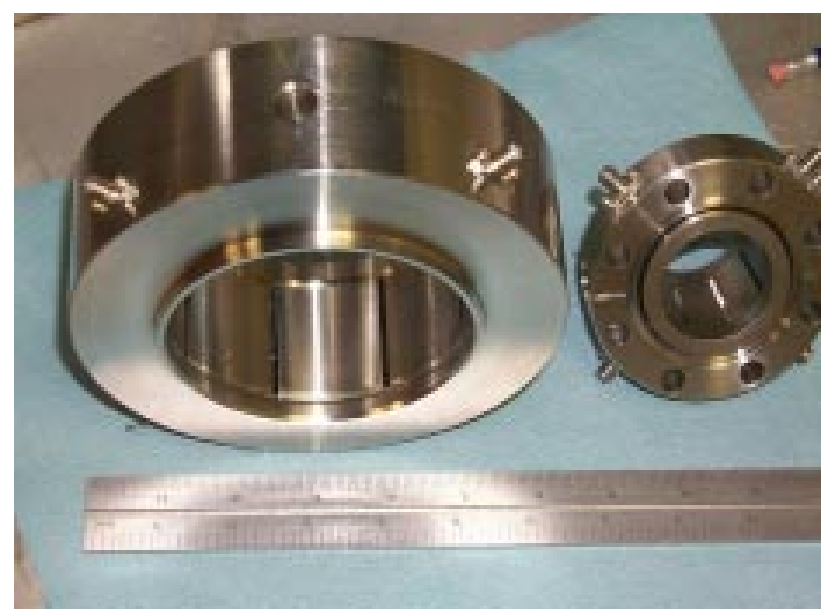

Fig. 1

\section{SIGNAL PROCESSOR}

The signal processor we used is simple and straightforward. The commercially available BPM electronic modules from a company named Bergoz [3] are employed. Each module is a 4"x6" printed circuit board, which accepts the 4 pick-up signals and gives the $\mathrm{X} / \mathrm{Y}$ output directly. Each BPM has its own electronic module. The amplifier gain of this module is set at $0.2 \mathrm{~V}$ output for $1 \%$ of tested signal difference. The 54 BPMs around the storage ring are divided into 4 divisions: Northwest, Southwest, Northeast, and Southeast. There are four crates located at each corner inside of the ring. Each crate is 17 inches wide, 11 inches high and 9 inches deep. The crate can hold up to 16 modules. High quality coaxial cables are used to connect each BPM pick-up electrode to the signal input at the crate. The 1/4" Heliax coaxial cables were selected to connect the straight section BPM because of its low loss $(2.4 \mathrm{~dB} / 100 \mathrm{ft}$ at $200 \mathrm{MHz})$ and good shielding, since the straight section BPM has lower sensitivity and the cables are longer. The relatively shorter cables for arc BPM are RG223 for economy reasons. Among the 54 BPMs, 34 of them have been connected to Bergoz electronic modules. From each Bergoz's module, there are 3 output signals (X, Y and sum) sent to a VME crate for computer readout. The VME crate is located at west-center of the ring where the EPICS system is connected. 


\section{SYSTEM PERFORMANCE}

The whole BPM system started to operate in September 1998. At the beginning, all the beam position data were fluctuated over a range of $5-15 \%$. Observations revealed that the $\mathrm{X} / \mathrm{Y}$ output signals had strong $\mathrm{AC}$ components and some of them were $60 \mathrm{HZ}$ related $\mathrm{AC}$ noises. It was contributed mainly by the magnet power supplies. The signal conditioning boards purchased from VMIC Company have been added to reduce the AC noises. Its function is to provide a low pass filter with a bandwidth of $3 \mathrm{~dB}$ attenuation at $4 \mathrm{HZ}$. With the conditioning boards, the fluctuation has been reduced to $+/-25$ micrometers over a short period (a few minutes) of time.

The system has a beam current resolution of about $1 \mathrm{~mA}$. When stored beam currents are below $1 \mathrm{~mA}$, the beam position data becomes current dependent. However, most of our ring operations are not at the low current region and we do not expect this to be a significant problem for us. The beam position dataes are currently delivered at a rate of $2 \mathrm{HZ}$. A sophisticated display tool (ADT) from APS is used for beam orbit displaying.

Overall, our BPM system is still in the process of being commissioned. Our future goals are:

1) Using the beam to calibrate the BPM offset related to nearby magnet

2) Using the beam position data to correct beam orbit (locally and globally)

3) Developing a slow orbit feedback system to correct the orbit drifting

4) Establish a fast (up to $120 \mathrm{HZ}$ ) beam orbit control feedback system.

\section{ACKNOWLEDGEMENT}

Authors would like to thank J. L. Pellegrin at SLAC and Jim Hinkson at LBL for the constructive discussions. The consulting with them has been very helpful for the work we did here.

\section{REFERENCES}

[1] V. N. Litvinenko, et al., "Commissioning of the Duke Storage Ring", Proceedings of 1995 Particle Accelerator Conference, Dallas, TX May 1-5, V. I, P. 213.

[2] J. Hinkson, "Advanced Light Source Beam Position Monitor", Conference Proceedings NO. 252, Accelerator Instrumentation Third Annual Workshop, Newport News, VA 1991 P. 21.

[3] Klaus B. Unser, "New Generation Electronics Applied to Beam Position Monitors", Bergoz Precision Beam Instrumentation, F-01170 Crozet, France. 